\title{
Using Existing Biodiversity Data to Prioritize Survey Areas in Atlas Projects
}

\author{
Laurens Sparrius * \\ ‡ FLORON Plant Conservation Netherlands, Nijmegen, Netherlands
}

Corresponding author: Laurens Sparrius (sparrius@floron.nl)

Received: 09 Jun 2019 | Published: 13 Jun 2019

Citation: Sparrius L (2019) Using Existing Biodiversity Data to Prioritize Survey Areas in Atlas Projects. Biodiversity Information Science and Standards 3: e37002. https://doi.org/10.3897/biss.3.37002

\begin{abstract}
Species distribution data is often collected in local and regional atlas projects, in which data is collected in grid squares to ensure optimal data coverage. Nowadays, many different data sources are available, and atlas projects often start with a large number of observations already being available. We show how existing biodiversity data can be used to determine spatial gaps in atlas projects. An citizen science atlas project for vascular plants in the Netherlands shows how existing data are is used to prioritize grid squares with outdated or missing surveys, and grid squares that should be searched for rare and declining species that have been reported in the past. Data from citizen science projects and professional flora surveys are combined in the National Database of Flora and Fauna (www.ndff.nl), which includes the use of data standards and extensive metadata on data collection protocols. This nearly complete database is used in citizen science projects to prioritize the survey of gaps. The concept of a continuously updated atlas scheme is established. Data can be use for several purposes, providing data for the national Red List, as well as reports on protected species, ecological studies, and the eradication of invasive species.
\end{abstract}

\section{Keywords}

citizen science, metadata, data use 
2

Sparrius L

Presenting author

Laurens B. Sparrius

Presented at

Biodiversity_Next 2019 